\title{
Systematic Approach to Compute the Vibrational Energy Levels of Diatomic Molecules
}

\author{
Mohammed Janati Idrissi, Abdelaziz Fedoul, Salaheddine Sayouri \\ Laboratory of Applied Physics Informatics and Statistics, Faculty of Sciences, Dhar Al Mahraz, University of Sidi Mohamed Ben \\ Abdellah, Fez, Morocco \\ Email:afedoul@yahoo.fr, ssayouri@gmail.com, jan123med@gmail.com
}

How to cite this paper: Idrissi, M.J., Fedoul, A. and Sayouri, S. (2020) Systematic Approach to Compute the Vibrational Energy Levels of Diatomic Molecules. Journal of Applied Mathematics and Physics, 8, 2463-2474.

https://doi.org/10.4236/jamp.2020.811182

Received: October 10, 2020

Accepted: November 21, 2020

Published: November 24, 2020

Copyright $\odot 2020$ by author(s) and Scientific Research Publishing Inc. This work is licensed under the Creative Commons Attribution International License (CC BY 4.0).

http://creativecommons.org/licenses/by/4.0/

\begin{abstract}
In continuation of our previous paper of the anharmonic potentials analysis through the Floquet representation, we performed in this work a systematic calculation of the diatomic vibrational energy levels as well as the corresponding wave functions. The solution of Schrödinger equation according to Morse potential, which is a suitable model to describe the diatomic vibrational spectra, has been introduced; thus the explicit formulas to the second order have been established. As an illustration, the dissociation energies of some molecules species (i.e. $\mathrm{ScN}, \mathrm{LiH}, \mathrm{Cl}_{2}$ and $\mathrm{NO}$ ) have been computed, as well as the wave functions and the corresponding probability densities, relating to the $(\mathrm{ScN})$ molecule have been represented. Comparisons of our results with those of literature have been made.
\end{abstract}

\section{Keywords}

Morse Potential, Diatomic Molecule, Dissociation, Birge-Sponer

\section{Introduction}

In view of the importance that has the harmonic, pseudo-harmonic and anharmonic oscillators in various fields of physics, they have been extensively used for solving most of physical problems. To this end, significant efforts are devoted to determining the general formulas of vibration-rotation energy spectra and wave functions for the molecular systems. The simple harmonic oscillator is a typical model, and a major tool in understanding many physical phenomena. In particular, the introduction of this model in quantum mechanics theory plays a fundamental role in the mathematical formalism of this theory, and it is one of the 
exactly solvable potentials of the Schrödinger equation [1] [2]. However, most realistic physical phenomena rely directly on deviation from the harmonic shape, which must be modeled by the addition of small non harmonic terms. The pseudo-harmonic oscillator pattern is an example of a realistic problem and it is considered as intermediate between harmonic and anharmonic potentials. The main characteristic of this potential is that it allows obtaining exact solutions of the Schrodinger equation for an arbitrary rotational quantum number, and leads to the calculation of the molecular vibration-rotation energy spectra in chemical physics [3] [4] [5] [6] [7]. Nevertheless, in order to restore the agreement of the theory with the experiments, the inclusion of the intrinsic anharmonic effects is fundamental for realistic systems. In general, it is impossible to find an exact solution to such systems, but one can apply conventional approximate methods usually presented in most standard textbooks and research papers [1] [2] [6] [7]. In this regard, modeling the atomic potential is one of the most important parts of molecular dynamics. Several anharmonic functions have been developed and applied to solve the Schrödinger equation of these systems, among them, we cite Dunham [8] and Pöschl-Teller [9]. In particular, the well-known Morse potential function is a more reasonable and realistic model [10] for these systems. In this regard, the Morse potential model provides an accurate description of the observed vibrational energy spectra of diatomic molecules, and has great popularity with chemists.

Our motivation is to continue exploring the principal formulas given in our previous paper of the analysis of anharmonic potentials through the Floquet representation [11], where some general results relating to the Morse potential have been established. The aim of this work is to perform the calculations used in Subsection 3.3, and to establish the explicit wave function to the second order of approximation [12]. These are realized for the following diatomic molecules ( $\mathrm{ScN}, \mathrm{LiH}, \mathrm{Cl}_{2}$ and $\mathrm{NO}$ ), to find the finite number of bound states and the corresponding dissociation energy as well as the wave functions. Besides, to clarify and confirm our results, we have also made the calculations, according to the graphical Birge-Sponer plot [13] to find again the obtained results; for the above-mentioned molecules.

The organization of the present paper is as follows: In Section 2 we give the outline of the theoretical context and the basic equations. In Section 3 we apply the analytic expressions to obtain numerical results and we give comparison. Section 4 presents the discussion. Section 5 contains the conclusions.

\section{Theoretical Background and Basic Equations}

The anharmonic Morse potential is frequently used to describe the vibrational spectra of diatomic molecules. Its analytical expression is such as [10] [14],

$$
V(q)=D_{e}\left(1-\mathrm{e}^{-\rho q}\right)^{2}
$$

where $D_{e}$ is a parameter that controls the depth of the potential well, $\rho$ is a 
parameter that measures the curvature of the potential well (width of attraction), and $q$ is the variation of interatomic distance with respect to the equilibrium distance.

Let us do the Taylor development of the exponential term in $V(q)$ to fourth order around $q=0$. Collecting the coefficients of the obtained series expansion gives,

$$
V(q)=D_{e} \rho^{2} q^{2}-D_{e} \rho^{3} q^{3}+\frac{7}{12} D_{e} \rho^{4} q^{4}
$$

The Morse oscillator Hamiltonian of a diatomic molecule of reduced mass $\mathrm{m}$ is given by,

$$
H_{M}=\frac{p^{2}}{2 m}+V(q)=\frac{p^{2}}{2 m}+D_{e} \rho^{2} q^{2}-D_{e} \rho^{3} q^{3}+\frac{7}{12} D_{e} \rho^{4} q^{4}
$$

Let us make the changes of the constant factors in the following notations,

$$
\begin{gathered}
\omega_{0}=\rho \sqrt{\frac{2 D_{e}}{m}} \\
\mu_{1}=-\frac{D_{e} \rho^{3}}{m \omega_{0}^{2}} \sqrt{\frac{\hbar}{m \omega_{0}}} \\
\mu_{2}=\frac{7}{12} \frac{D_{e} \rho^{4} \hbar}{m^{2} \omega_{0}^{3}}
\end{gathered}
$$

where $\omega_{0}$ is the vibrational constant of the diatomic molecule.

Then we find the anharmonic oscillator which Hamiltonian is such that,

$$
H(\hat{q})=\frac{p^{2}}{2 m}+\frac{1}{2} m \omega_{0}^{2} q^{2}+\mu_{1} \hbar \omega_{0} \hat{q}^{3}+\mu_{2} \hbar \omega_{0} \hat{q}^{4}
$$

where $\hat{q}=\sqrt{\frac{m \omega_{0}}{\hbar}} q$ and where $\mu_{1} \hbar \omega_{0} \hat{q}^{3}$ and $\mu_{2} \hbar \omega_{0} \hat{q}^{4}$ are the cubic and quadric anharmonic perturbations with amplitudes $\mu_{1}$ and $\mu_{2}$ respectively.

According to Equations (3) and (4), we have the similar situation given by the cubic and quadric anharmonic oscillators, for which the Hamiltonian is also given by Equation (5).

Using Equations (24) and (35) of our paper [11], and replacing the constant factors of Equation (4), one can obtain the quasi-energy ${ }^{(2 a)} E_{n}$ up to second ameliorated order, such as,

$$
\begin{aligned}
{ }^{(2 a)} E_{n}= & \hbar \omega_{0}\left(n+\frac{1}{2}\right)+\frac{7}{16} \frac{D_{e} \rho^{4} \hbar^{2}}{m^{2} \omega_{0}^{2}}\left(2 n^{2}+2 n+1\right)-\frac{15}{4} \frac{D_{e}^{2} \rho^{6} \hbar^{2}}{m^{3} \omega_{0}^{4}}\left(n^{2}+n+\frac{11}{30}\right) \\
& -\frac{49}{1152} \frac{D_{e}^{2} \rho^{8} \hbar^{3}}{m^{4} \omega_{0}^{5}}\left(34 n^{3}+51 n^{2}+59 n+21\right)
\end{aligned}
$$

We emphasize that the Morse potential anharmonicity effect appears clearly to the second order calculation and that it affects the high vibrational energy levels.

Let us remark that, 


$$
\begin{aligned}
& \qquad \begin{array}{c}
2 n^{2}+2 n+1=2\left(n+\frac{1}{2}\right)+\frac{1}{2} \\
\left(n^{2}+n+\frac{11}{30}\right)=\left(n+\frac{1}{2}\right)^{2}+\frac{7}{60} \\
\text { and }\left(34 n^{3}+51 n^{2}+59 n+21\right)=34\left(n+\frac{1}{2}\right)^{3}+\frac{67}{2}\left(n+\frac{1}{2}\right)
\end{array}
\end{aligned}
$$

Then Equation (6) can be written in term of $\left(n+\frac{1}{2}\right)$ such as,

$$
\begin{aligned}
{ }^{(2 a)} E_{n}= & \hbar \rho \sqrt{\frac{2 D_{e}}{m}}\left[1-\frac{3283}{18432} \frac{\hbar^{2} \rho^{2}}{m D_{e}}\right]\left(n+\frac{1}{2}\right)-\frac{\hbar^{2} \rho^{2}}{2 m}\left(n+\frac{1}{2}\right)^{2} \\
& -\frac{833}{2304} \frac{\hbar^{3} \rho^{3}}{m^{2}} \sqrt{\frac{m}{2 D_{e}}}\left(n+\frac{1}{2}\right)^{3}
\end{aligned}
$$

Since the experiments data neglected the $\left(n+\frac{1}{2}\right)^{3}$ term, we can read Equation (7) as,

$$
{ }^{(2 a)} E_{n}=\hbar \rho \sqrt{\frac{2 D_{e}}{m}}\left[1-\frac{3283}{18432} \frac{\hbar^{2} \rho^{2}}{m D_{e}}\right]\left(n+\frac{1}{2}\right)-\frac{\hbar^{2} \rho^{2}}{2 m}\left(n+\frac{1}{2}\right)^{2}
$$

Taking the derivative with respect to $n$ of Equation (8) gives,

$$
\frac{\mathrm{d}^{(2 a)} E_{n}}{\mathrm{~d} n}=\hbar \rho \sqrt{\frac{2 D_{e}}{m}}\left[1-\frac{3283}{18432} \frac{\hbar^{2} \rho^{2}}{m D_{e}}\right]-\frac{\hbar^{2} \rho^{2}}{m}\left(n+\frac{1}{2}\right)
$$

Equations (8) and (9) play the basic role in our analysis of the diatomic vibrational energy.

Let us note that the energies levels spacing will decrease with increasing values of $n$, therefore when $\frac{\mathrm{d}^{(2 a)} E_{n}}{\mathrm{~d} n}=0$, the vibrational quantum number takes the maximum value $n_{\max }$, i.e. the value for the vibrational quantum number where dissociation occurs, which allows us to determine the dissociation energy of the diatomic molecule.

Let us also note that the function $\frac{\mathrm{d}^{(2 a)} E_{n}}{\mathrm{~d} n}$ versus $\left(n+\frac{1}{2}\right)$ decreases as a linear function of the variable $\left(n+\frac{1}{2}\right)$.

Using the expressions of the evolution operator, to the second order given by Equations (3) and (4b) [12], leads to obtain the second ameliorated solutions terms of quantum states, then we can express the wave-functions of the system as follows,

$$
{ }^{(2 a)} \psi_{n}(\hat{q})=\left(\frac{m \omega_{0}}{\hbar \pi}\right)^{\frac{1}{4}} \frac{\mathrm{e}^{-\frac{\hat{q}^{2}}{2}}}{\sqrt{2^{n} n !}} \sum_{j=-8}^{n+8} K_{n+j}^{n} H_{n+j}(\hat{q})
$$

where $H_{n}(\hat{q})$ are the Hermite polynomials. 
And where the different coefficients $K_{n+j}^{n}$ are given by,

$$
\begin{aligned}
& K_{n-8}^{n}=\frac{\mu_{2}^{2}}{32} \frac{n !}{(n-8) !} \\
& K_{n-7}^{n}=\frac{\sqrt{2}}{192} \mu_{1} \mu_{2} \frac{n !}{(n-7) !} \\
& K_{n-6}^{n}=\frac{1}{2}\left[\frac{\mu_{1}^{2}}{9}+\left(n-\frac{11}{6}\right) \frac{\mu_{2}^{2}}{2}\right] \frac{n !}{(n-6) !} \\
& K_{n-5}^{n}=\frac{17}{24} \mu_{1} \mu_{2}\left(n-\frac{104}{85}\right) \frac{n !}{(n-5) !} \\
& K_{n-4}^{n}=\frac{1}{4}\left[\mu_{2}+\left(2 n-\frac{3}{2}\right) \mu_{1}^{2}+(n-1)(2 n-7) \mu_{2}^{2}\right] \frac{n !}{(n-4) !} \\
& K_{n-3}^{n}=\left[\frac{\mu_{1}}{3}+\left(21 n^{2}-74 n+29\right) \frac{\mu_{1} \mu_{2}}{16}\right] \frac{n !}{(n-3) !} \\
& K_{n-2}^{n}=\frac{1}{2}\left[(2 n-1) \mu_{2}+\left(7 n^{2}-19 n+1\right) \frac{\mu_{1}^{2}}{4}-\left(4 n^{3}+60 n^{2}-16 n+15\right) \frac{\mu_{2}^{2}}{8}\right] n(n-1) \\
& K_{n-1}^{n}=\frac{1}{2}\left[3 n \mu_{1}-\left(81 n^{3}+834 n^{2}+203 n+274\right) \frac{\mu_{1} \mu_{2}}{48}\right] n \\
& K_{n}^{n}=1+\frac{\mu_{1}^{2}}{144}(2 n+1)\left(82 n^{2}+82 n+87\right)+\frac{1}{256} \mu_{2}^{2}\left(65 n^{4}+130 n^{3}+487 n^{2} 422 n+156\right) \\
& K_{n+1}^{n}=\frac{1}{4}\left[-3(n+1) \mu_{1}+\left(-81 n^{3}+591 n^{2}+1222 n+816\right) \frac{\mu_{1} \mu_{2}}{48}\right] \\
& K_{n+2}^{n}=\frac{1}{8}\left[-(2 n+3) \mu_{2}+\left(7 n^{2}+33 n+27\right) \frac{\mu_{1}^{2}}{4}+\left(4 n^{3}+48 n^{2}+142 n+87\right) \frac{\mu_{2}^{2}}{8}\right] \\
& K_{n+3}^{n}=\frac{1}{8}\left[-\frac{\mu_{1}}{3}+\left(21 n^{2}+116 n+124\right) \frac{\mu_{1} \mu_{2}}{16}\right] \\
& K_{n+4}^{n}=\frac{1}{64}\left[-\mu_{2}+\left(2 n+\frac{7}{2}\right) \mu_{1}^{2}+\left(2 n^{2}+13 n+13\right) \mu_{2}^{2}\right] \\
& K_{n+5}^{n}=\frac{17}{768}\left(n+\frac{108}{85}\right) \mu_{1} \mu_{2} \\
& K_{n+6}^{n}=\frac{1}{128}\left[\frac{\mu_{1}^{2}}{9}+\left(n+\frac{17}{6}\right) \frac{\mu_{2}^{2}}{2}\right] \\
& K_{n+7}^{n}=\frac{\mu_{1} \mu_{2}}{1536} \\
& K_{n+8}^{n}=\frac{\mu_{2}^{2}}{8192}
\end{aligned}
$$

As illustration the first three states are given below.

Ground state $(n=0)$ 


$$
\begin{array}{cl}
{ }^{(2 a)} \psi_{0}(\hat{q})=\frac{1}{23040}\left(\frac{m \omega_{0}}{\hbar \pi}\right)^{\frac{1}{4}} \mathrm{e}^{-\frac{\hat{q}^{2}}{2}} \sum_{j=0}^{8} C_{j}^{0} \hat{q}^{j} \\
C_{0}^{0}=45\left(512+288 \mu_{2}-272 \mu_{1}^{2}-407 \mu_{2}^{2}\right), & C_{1}^{0}=-720\left(32+27 \mu_{2}\right) \mu_{1} \\
C_{2}^{0}=360\left(-48 \mu_{2}+88 \mu_{1}^{2}+129 \mu_{2}^{2}\right), & C_{3}^{0}=480\left(261 \mu_{2}-16\right) \mu_{1} \\
C_{4}^{0}=120\left(-48 \mu_{2}+88 \mu_{1}^{2}-81 \mu_{2}^{2}\right), & C_{5}^{0}=576 \mu_{1} \mu_{2} \\
C_{6}^{0}=160\left(8 \mu_{1}^{2}+39 \mu_{2}^{2}\right), & C_{7}^{0}=1920 \mu_{1} \mu_{2} \\
C_{8}^{0}=720 \mu_{2}^{2} &
\end{array}
$$

First excited state $(n=1)$

$$
\begin{aligned}
{ }^{(2 a)} \psi_{1}(\hat{q})=\frac{\sqrt{2}}{23040}\left(\frac{m \omega_{0}}{\hbar \pi}\right)^{\frac{1}{4}} \mathrm{e}^{-\frac{\hat{q}^{2}}{2}} \sum_{j=0}^{9} C_{j}^{1} \hat{q}^{j} & C_{1}^{1}=-45\left(1488 \mu_{1}^{2}+3023 \mu_{2}^{2}-1440 \mu_{2}-512\right) \\
C_{0}^{1}=-120\left(2065 \mu_{2}-384\right) \mu_{1}, & C_{2}^{1}=120\left(568 \mu_{1}^{2}+537 \mu_{2}^{2}-240 \mu_{2}\right) \\
C_{2}^{1}=-240\left(833 \mu_{2}+192\right) \mu_{1}, & C_{3}^{1} \\
C_{4}^{1}=960\left(207 \mu_{2}-8\right) \mu_{1}, & C_{5}^{1}=120\left(152 \mu_{1}^{2}-21 \mu_{2}^{2}-48 \mu_{2}\right) \\
C_{6}^{1}=10176 \mu_{1} \mu_{2}, & C_{7}^{1}=160\left(8 \mu_{1}^{2}+57 \mu_{2}^{2}\right) \\
C_{8}^{1}=1920 \mu_{1} \mu_{2}, & C_{9}^{1}=720 \mu_{2}^{2}
\end{aligned}
$$

Second excited state $(n=2)$

$$
\begin{array}{ll}
{ }^{(2 a)} \psi_{2}(\hat{q})=\frac{1}{46080}\left(\frac{m \omega_{0}}{\hbar \pi}\right)^{\frac{1}{4}} \mathrm{e}^{-\frac{\hat{q}^{2}}{2}} \sum_{j=0}^{10} C_{j}^{2} \hat{q}^{j} \\
C_{0}^{2}=-45\left(2480 \mu_{1}^{2}+5041 \mu_{2}^{2}+1440 \mu_{2}+512\right), & C_{1}^{2}=-240\left(9349 \mu_{2}-1632\right) \mu_{1} \\
C_{2}^{2}=-90\left(3632 \mu_{1}^{2}+7139 \mu_{2}^{2}-3936 \mu_{2}-512\right), & C_{3}^{2}=-960\left(1823 \mu_{2}+136\right) \mu_{1} \\
C_{4}^{2}=120\left(1528 \mu_{1}^{2}+75 \mu_{2}^{2}-624 \mu_{2}\right), & C_{5}^{2}=768\left(663 \mu_{2}-20\right) \mu_{1} \\
C_{6}^{2}=80\left(632 \mu_{1}^{2}-141 \mu_{2}^{2}-144 \mu_{2}\right), & C_{7}^{2}=37632 \mu_{1} \mu_{2} \\
C_{8}^{2}=80\left(32 \mu_{1}^{2}+29 \mu_{2}^{2}\right), & C_{9}^{2}=3840 \mu_{1} \mu_{2} \\
C_{10}^{2}=1440 \mu_{2}^{2} &
\end{array}
$$

\section{Calculation Results}

In attempt to illustrate the established Equations (8) and (9), we performed the numerical computation using the Maple software to obtain the values of the parameters, corresponding to the previously mentioned diatomic molecules.

Solving the following equation: $\frac{\mathrm{d}^{(2 a)} E_{n}}{\mathrm{~d} n}=0$, round down to the nearest integer, leads to find the maximum vibrational quantum number $n_{\max }$ associated with the highest bound state energy level such as, 


$$
n_{\max }=\frac{\sqrt{2 D_{e} m}}{\hbar \rho}-\frac{3283 \sqrt{2}}{18432} \frac{\hbar \rho}{\sqrt{D_{e} m}}-\frac{1}{2}
$$

Therefore, substituting the obtaiend value $n_{\max }$ in Equation (8), gives the theoretical dissociation energy $D_{e}^{\text {th }}$ connected to the equilibrium position (i.e.the depth of the Morse potential well) of the molecule.

We have chosen the molecules ( $\mathrm{ScN}, \mathrm{LiH}, \mathrm{Cl}_{2}$ and $\mathrm{NO}$ ) whose parameters are given in Table 1.

The calculated values of the maximum quantum numbers, the theoretical dissociation energy and the dissociation energy with respect to the zero point level corresponding to $\left(\mathrm{ScN}, \mathrm{LiH}, \mathrm{Cl}_{2}\right.$ and $\left.\mathrm{NO}\right)$ molecules are given in Table 2 .

Next, from Equation (9) we can compute the differences between any two successive energies levels: $\Delta E={ }^{(2 a)} E_{n}-{ }^{(2 a)} E_{n-1}$, which can be written as follows,

$$
\Delta E=\frac{\mathrm{d}^{(2 a)} E_{n}}{\mathrm{~d} n}+\frac{\hbar^{2} \rho^{2}}{2 m}=\hbar \rho \sqrt{\frac{2 D_{e}}{m}}\left[1-\frac{3283}{18432} \frac{\hbar^{2} \rho^{2}}{m D_{e}}\right]+\frac{\hbar^{2} \rho^{2}}{2 m}-\frac{\hbar^{2} \rho^{2}}{m}\left(n+\frac{1}{2}\right)
$$

Thence the graphics of the Birge-Sponer plots, corresponding to $\mathrm{ScN}, \mathrm{LiH}$, and $\mathrm{Cl}_{2}$ and $\mathrm{NO}$ molecules are given respectively in Figure 1.

In Figure 2 and Figure 3, we present the plots of the wave functions ${ }^{(2 a)} \psi_{n}(\hat{q})$ versus $\hat{q}$, corresponding to the first six $(n=0,1,2,3,4,5)$ levels, and the probability densities of the wave-packet $\left(\left|{ }^{(2 a)} \psi_{n}(\hat{q})\right|^{2}\right)$ in the cases where the number states are $n=0$ and $n=5$ respectively, for the $(\mathrm{ScN})$ molecule.

\section{Discussion}

Table 1. The molecules parameters.

\begin{tabular}{cccc}
\hline Molecule & $\rho\left(10^{10} \mathrm{~m}^{-1}\right)$ & $m(\mathrm{amu})$ & $D_{e}(\mathrm{eV})$ \\
\hline $\mathrm{ScN}[3]$ & 1.50680 & 10.682771 & 4.56 \\
$\mathrm{LiH} \mathrm{[4]}$ & 1.128 & 0.8801221 & 2.515287 \\
$\mathrm{Cl}_{2}[5]$ & 2.0087 & 17.608328 & 2.513926 \\
$\mathrm{NO}[5]$ & 2.7534 & 7.521478 & 6.613502 \\
\hline
\end{tabular}

Table 2. The maximum quantum numbers, theoretical dissociation energy and comparison.

\begin{tabular}{cccccc}
\hline & \multicolumn{3}{c}{ This work } & \multicolumn{2}{c}{ Literature } \\
\cline { 2 - 6 } Molecule & $n_{\max }$ & $D_{e}^{\text {th }}(\mathrm{eV})$ & $D_{0}^{\text {th }}(\mathrm{eV})$ & $n_{\max }$ & $D_{e}(\mathrm{eV})$ \\
\hline $\mathrm{ScN}$ & 101 & 4.5596 & 4.5147 & --- & $4.56[3]$ \\
$\mathrm{LiH}$ & 28 & 2.5128 & 2.426 & $29[4][15]$ & $2.515287[4][15]$ \\
& & & & $2.515267[3]$ \\
$\mathrm{Cl}_{2}$ & 72 & 2.5136 & 2.4790 & $72[6]$ & $2.513926[5]$ \\
$\mathrm{NO}$ & 55 & 6.6115 & 6.4940 & $55[6]$ & $6.613502[5]$ \\
\hline
\end{tabular}



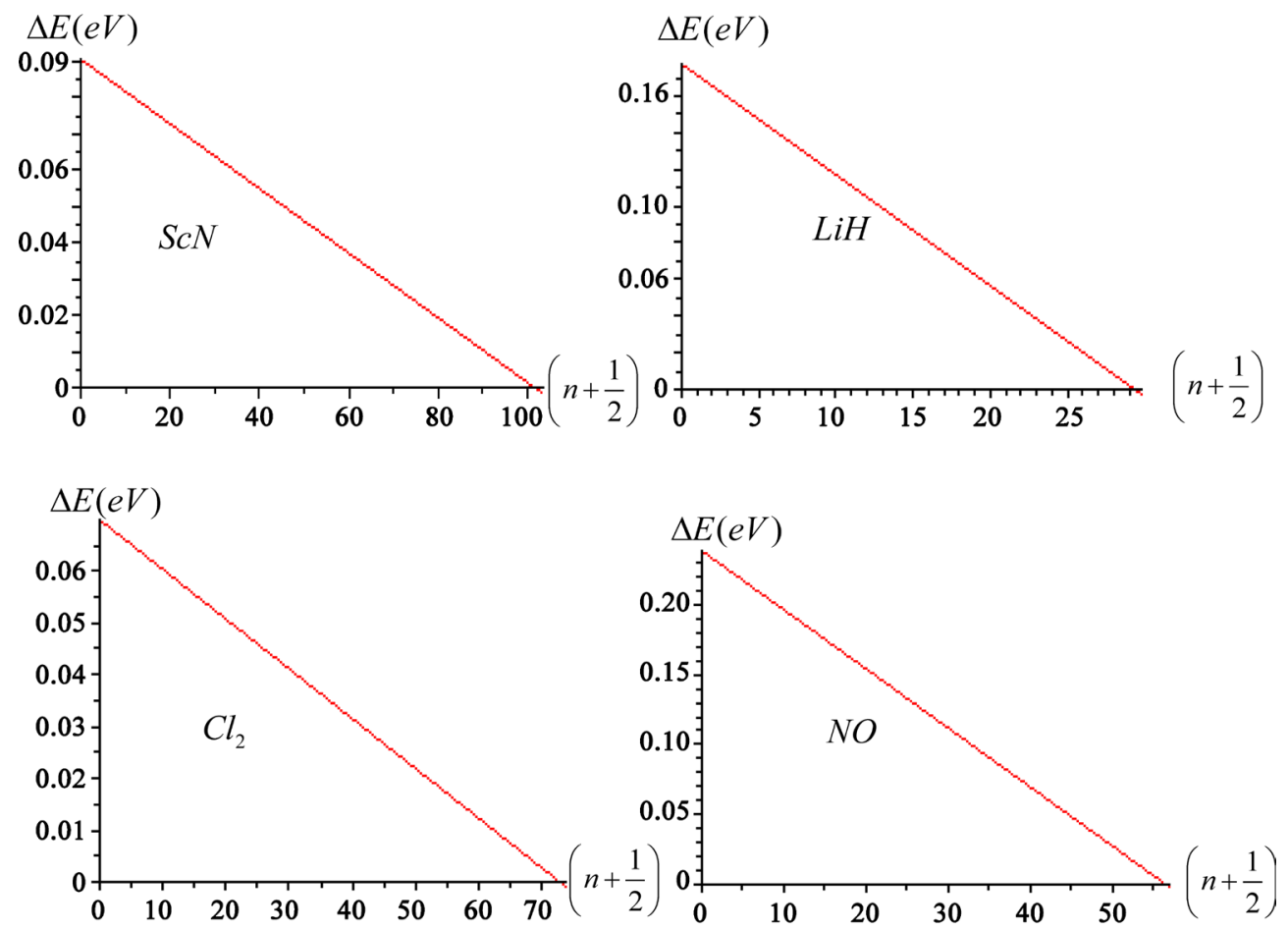

Figure 1. The variation of $\Delta E$ vs. $\left(n+\frac{1}{2}\right)$, of the $\left(\mathrm{ScN}, \mathrm{LiH}, \mathrm{Cl}_{2}\right.$ and $\left.\mathrm{NO}\right)$ molecules. For all graphics, the vertical axis is the energy between, two successif levels and the horizontal axis is $\left(n+\frac{1}{2}\right)$.
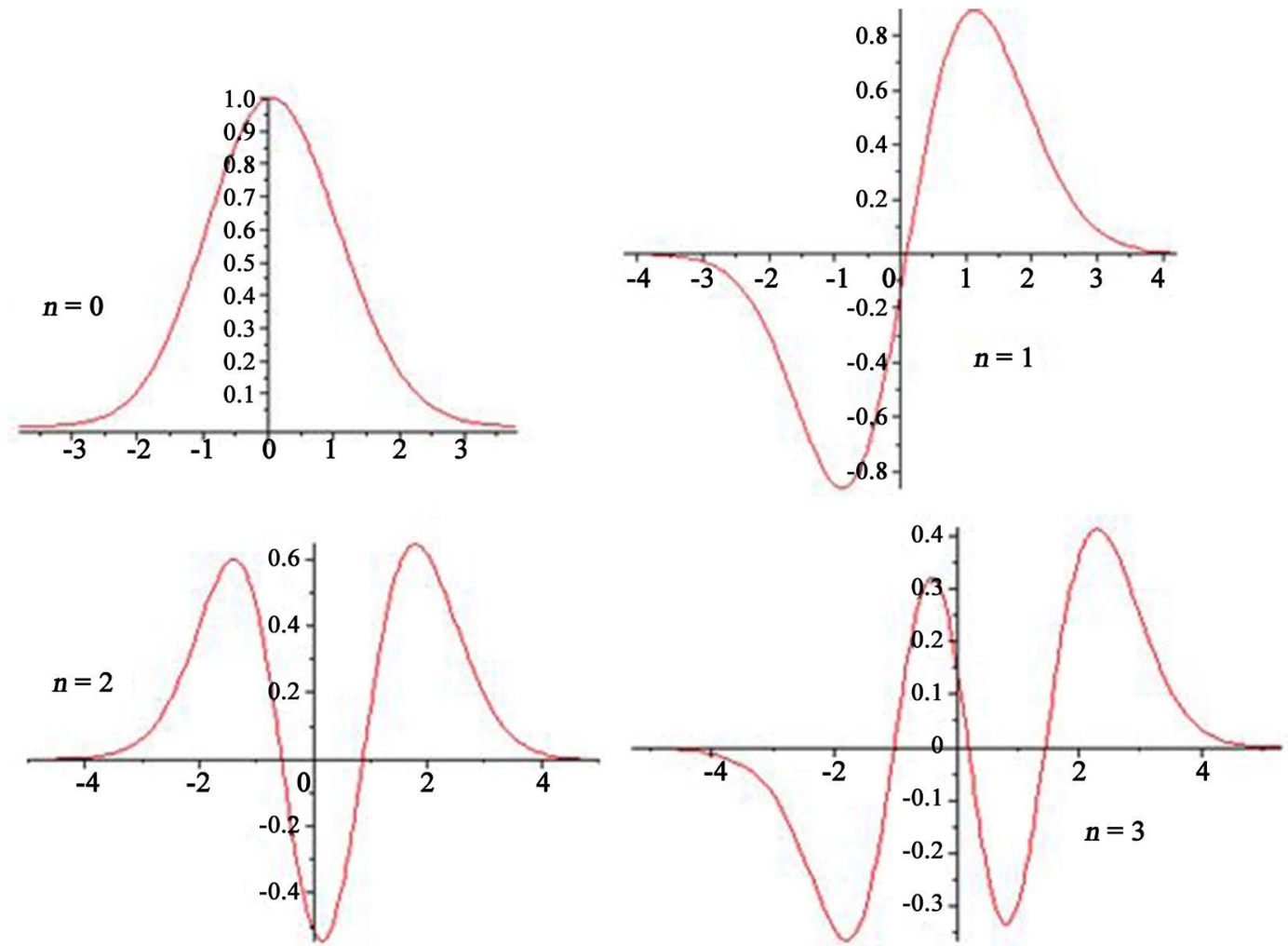

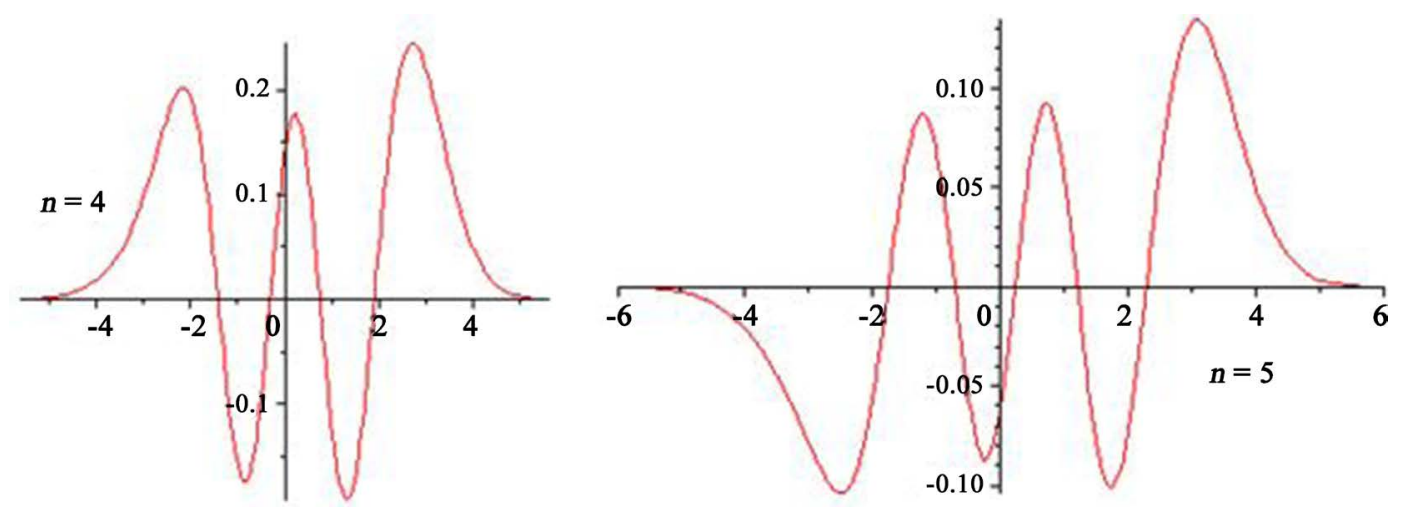

Figure 2. Plots of the wave functions relating to the $\mathrm{ScN}$ molecule. For all graphics $(n=1$ to $n=5)$, the vertical axis is the wave function $\left(\frac{\hbar \pi}{m \omega_{0}}\right)^{\frac{1}{4}}{ }^{(2 a)} \psi_{n}(\hat{q})$ and the horizontal axis is the $\hat{q}$.
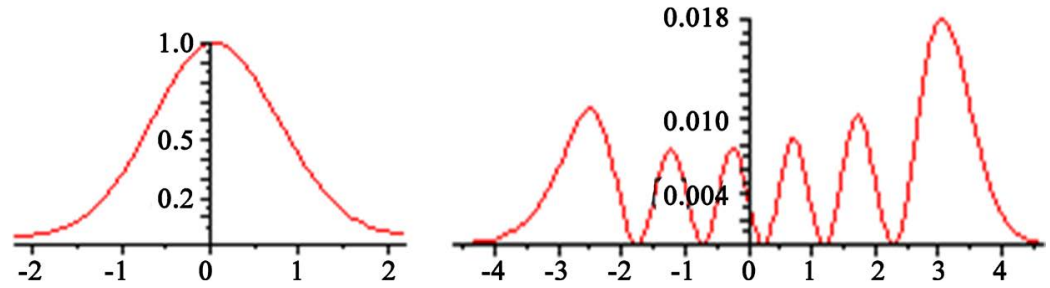

Figure 3. Plots of the probability densities relating to the $\mathrm{ScN}$ molecule. For all graphics ( $n$ $=0$ and $n=5)$, the vertical axis is the probability densities $\left(\left(\frac{\hbar \pi}{m \omega_{0}}\right)^{\frac{1}{2}}\left|(2 a) \psi_{n}(\hat{q})\right|^{2}\right)$ and the horizontal axis is the $\hat{q}$.

The main goal of our paper is to give an alternative way, to solve the quantum anharmonic oscillator problem [11] [12]. This non-perturbative processing is applied to determine the analytical expressions of the energy eigenvalues and eigenfunctions of the Morse potential.

The second order calculation of vibrational energy levels according to the Morse oscillator where performed in Equations (6) and (7). Therefore, Equation (7) is used for the numerical calculation, based on the parameter values of the molecules selected from Table 1 .

Table 2 shows the numerical results and some comparisons.

Note that the graphs of $\Delta E$ versus $\left(n+\frac{1}{2}\right)$ of Equation (15) are lines with negative slopes. Therefore the spacing $\Delta E$ between adjacent vibrational energy levels is a decreasing linear function of the variable $\left(n+\frac{1}{2}\right)$. Thus the plots of Figure 1 allowed us to find again the results cited in Table 2.

The value of $\left(n_{\max }+\frac{1}{2}\right)$ is the intersection of the curve with the abscissa axis, as well as integral of $\Delta E$ over $\left(n+\frac{1}{2}\right)$ from $\left(n=0\right.$ to $\left.n=n_{\max }\right)$ to ob- 
tain the area under the curve, gives the dissociation energy $D_{0}$. Then we can compute $D_{e}^{\text {th }}=D_{0}+{ }^{(2 a)} E_{0}$.

Figure 2 and Figure 3 show that all the pictures profile of the wave functions and the corresponding probability densities, present the regular nodal character. The first excited state has one node and the second excited state has two nodes and so on. But their peaks are not symmetrical, therefore, the harmonic vibrations of the molecule are degraded, this is due to the anharmonic effect of the Morse potential well.

\section{Conclusions}

In this work, we performed the calculations of the vibrational energy levels as well as the wave functions of the diatomic molecules, according to a systematic approach. Our computations were carried out using the parameters of the Morse potential of some molecules, namely $\left(\mathrm{ScN}, \mathrm{LiH}, \mathrm{Cl}_{2}\right.$ and $\mathrm{NO}$ ). Then, with the help of the Maple software, we have determined the value of the maximum vibrational quantum number $\left(n_{\max }\right)$ and the theoretical dissociation energy $D_{e}^{\text {th }}$ corresponding to the previous mentioned molecules.

Moreover, we also represented in Figure 1, the so-called Birge-Sponer plot, deduced from the established general formula (8), which shows that the difference $\Delta E$ between two successive vibrational energy levels decreases as a linear function of the variable $\left(n+\frac{1}{2}\right)$. This method allowed us as well to find again the values: $n_{\max }$ and $D_{e}^{\text {th }}$ of these molecules. From Table 1 and Table 2, it can be seen that the two procedures based on the general formula (8) agree well, when applying them to the previous diatomic molecules. In addition, the established fundamental expression of the wave functions, has been positively illustrated in Figure 2 with the six levels $(n=0,1,2,3,4,5)$ as well as the probability densities with ( $n=0$ and $n=5)$ representation for the $(\mathrm{ScN})$ diatomic molecule. Comparison of our results with those established by other authors reveals a good agreement and shows the accuracy of our approach. Moreover, it can be applied to all forms of potential.

We project in futures works to improve the calculations, where $V(q)$ will be developed with more higher power terms, and we will study the ro-vibrational energy of diatomic molecules, according to the Deng-Fan potential.

Finally, we would like to point out that our study could be a useful tool for future research students to understand the quantum dynamics of anharmonic systems.

\section{Conflicts of Interest}

The authors declare no conflicts of interest regarding the publication of this paper.

\section{References}

[1] Cohen-Tannoudji, C., Diu, B. and Laloë, F. (1973) Mécanique Quantique T2. Her- 
mann, Paris, 1083-1224.

[2] Flügge, S. (1994) Practical Quantum Mechanics. Springer-Verlag, New York, 68-84.

[3] Oyewumia, K.J., Falayea, B.J., Onatea, C.A., Oluwadareb, O.J. and Yahyac, W.A. (2014) Thermodynamic Properties and the Approximate Solutions of the Schrödinger Equation with the Shifted Deng-Fan Potential Model. Molecular Physics, 112, 127-141. https://doi.org/10.1080/00268976.2013.804960

[4] Ikhdair, S.M. (2012) Effective Schrödinger Equation with General Ordering Ambiguity Position-Dependent Mass Morse Potential. Molecular Physics, 110, 1415-1428. https://doi.org/10.1080/00268976.2012.656148

[5] Kunc, J.A. and Gordillo-Vázquez, F.J. (1997) Rotational-Vibrational Levels of Diatomic Molecules Represented by the Tietz-HuaRotating Oscillator. The Journal of Physical Chemistry A, 101, 1595-1602. https://doi.org/10.1021/jp962817d

[6] Gorbachev, Y.J., Gordillo-Vázquez, F.J. and Kunc, J.A. (1997) Diameters of Rotationally and Vibrationally Excited Diatomic Molecules. Physica A, 247, 108-120. https://doi.org/10.1016/S0378-4371(97)00389-0

[7] Rydberg, R. (1931) Graphic Representation of Results of Band Spectroscopy. Zeitschrift für Physik, 73, 376-385. https://doi.org/10.1007/BF01341146

[8] Dunham, J.L. (1929) Intensities in the Harmonic Band of Hydrogen Chloride. Physical Review, 34, 438-452. https://doi.org/10.1103/PhysRev.34.438

[9] Pöschl, G. and Teller, E. (1933) Bemerkungen zur Quantenmechanik des anharmonischen Oszillators. Zeitschrift für Physik, 83, 143-151. https://doi.org/10.1007/BF01331132

[10] Morse, P.M. (1929) Diatomic Molecules According to the Wave Mechanics. II. Vibrational Levels. Physical Review, 34, 57-64. https://doi.org/10.1103/PhysRev.34.57

[11] Janati Idrissi, M., Fedoul, A., Sayouri, S. and Amila, I. (2020) Anharmonic Potentials Analysis through the Floquet Representation. Journal of Applied Mathematics and Physics, 8, 184-195. https://doi.org/10.4236/jamp.2020.81014

[12] Janati Idrissi, M., Fedoul, A., Achkar, Y., Chatwiti, A. and Sayouri, S. (2011) Squeezing in Floquet States and Quasi-Energies of Harmonic Oscillator Driven by a Strong Periodic Field. African Journal of Mathematical Physics, 10, 21-30.

[13] Birge, R.T. and Sponer, H. (1926) The Heat of Dissociation of Non-Polar Molecules. Physical Review, 28, 259-283. https://doi.org/10.1103/PhysRev.28.259

[14] Sun, H. (2005) The Morse Potential Eigenenergy by the Analytical Transfer Matrix Method. Physics Letters A, 338, 309-314. https://doi.org/10.1016/j.physleta.2005.02.054

[15] Nasser, I., Abdelmonem, M.S., Bahlouli, H. and Alhaidari, A.D. (2007) The Rotating Morse Potential Model for Diatomic Molecules in the Tridiagonal J-Matrix Representation: I. Bound States. Journal of Physics B: Atomic, Molecular and Optical Physics, 40, 4245-4257. https://doi.org/10.1088/0953-4075/40/21/011 


\section{Nomenclatures}

$V(q)$ : Morse potential

$D_{e}$ : Dissociation energy

$\rho$ : Parameter that controls the width of attraction

$H_{M}$ : Morse oscillator Hamiltonian

$m$ : Reduced mass of the diatomic system

$\omega_{0}$ : Unperturbed oscillator frequency

$p$ : Impulsion operator

$q$ : Position operator

$\hbar$ : Reduced Planck's constant

${ }^{(2 a)} E_{n}$ : Quasi-energy to second order

n: Quantum number

$n_{\max }$ : Maximum quantum number

${ }^{(2 a)} \psi_{n}(\hat{q})$ : Wave-functions to second order

$H_{n}(\hat{q}):$ Hermite polynomials 